Écrire

l'histoire

\section{Écrire l'histoire}

Histoire, Littérature, Esthétique

\section{9 | 2019}

\section{L'historien et les langues}

\title{
Livres récents
}

Sélection d'ouvrages parus en 2018

Jacob Lachat

\section{OpenEdition}

Journals

Édition électronique

URL : http://journals.openedition.org/elh/1982

DOI : $10.4000 /$ elh. 1982

ISSN : 2492-7457

Éditeur

CNRS Éditions

\section{Édition imprimée}

Date de publication : 1 décembre 2019

Pagination : 247-253

ISBN : 978-2-271-12967-3

ISSN : 1967-7499

Référence électronique

Jacob Lachat, «Livres récents », Écrire l'histoire [En ligne], 19 | 2019, mis en ligne le 01 décembre 2019, consulté le 16 février 2021. URL : http://journals.openedition.org/elh/1982 ; DOI : https://doi.org/ 10.4000/elh.1982 


\section{Livres récents}

Sélection d'ouvrages parus en 2018 Rubrique établie par Jacob Lachat

\section{Éditions, rééditions et traductions}

Adorno Theodor W., Actualité de la philosophie et autres essais, traduit de l'allemand par Pierre Arnoux, Julia Christ, Georges Felten, Anne Le Goff, Florian Nicomède et Matthias Nicomède, sous la direction de Jacques-Olivier Bégot, postface de Jacques-Olivier Bégot, Paris, Rue d'Ulm.

Alembert Jean Le Rond d', Mélanges de littérature, d'histoire et de philosophie, édition de Martine Groult, Paris, Classiques Garnier, coll. «Lire le dix-huitième siècle ».

Althusser Louis, Écrits sur l'histoire 1963-1989, édition établie par Michael Goshgarian, Paris, PUF, coll. " Perspectives critiques ».

Armenteros Carolina, L'Idée française de l'histoire. Joseph de Maistre et sa postérité, 1794-1854, nouvelle édition avec une préface de Philippe Barthelet, Paris, Classiques Garnier, coll. «Classiques jaunes ».

Aron Jean-Paul, Fouque Antoinette, Le Brun Annie, Lipovetsky Gilles, NorA Pierre, SARtre Jean-Paul, Sollers Philippe (dir.), Commémorer Mai 68 ?, Paris, Gallimard, coll. « Folio ».

Benjamin Walter, Karl Kraus, traduit de l'allemand par Marion Maurin et Antonin Wiser, Paris, Allia.

Bergson Henri, Histoire des théories de la mémoire. Cours au Collège de France 1903-1904, édition établie par Arnaud François, Paris, PUF.

Boucheron Patrick, Faire profession d'historien, Paris, Éditions du Seuil, coll. « Points ».

Canetti Elias, Le Livre contre la mort, Paris, Albin Michel, coll. «Les grandes traductions ».

Dosse François, Castoriadis, une vie, Paris, La Découverte.

Flavius JosèPHe, Contre Apion, texte établi et annoté par Théodore Reinach, traduction par Léon Blum, introduction et notes complémentaires par Sylvie Anne Goldberg, Paris, Les Belles Lettres.

Fondane Benjamin, Devant l'histoire, textes réunis et présentés par Monique Jutrin, Paris, Éditions de l'Éclat, coll. « Philosophie imaginaire ».

Foucault Michel, Histoire de la sexualité IV. Les aveux de la chair, édition de Frédéric Gros, Paris, Gallimard, coll. « Bibliothèque des Histoires ». 
Haneda Masashi, Toward Creation of a New World History, translated by Noda Makito, Tokyo, Japan Publishing Industry Foundation for Culture.

Hartog François, Partir pour la Grèce, édition revue et augmentée, Paris, Flammarion. Hugo Victor, Les Châtiments, nouvelle édition par Jean-Marc Hovasse, Paris, Flammarion, coll. «GF ».

—, Notre-Dame de Paris, édition établie par Marius-François Guyard, Paris, Classiques Garnier.

—, Les Misérables, édition d'Henri Scepi avec la collaboration de Dominique Moncond'huy, Paris, Gallimard, coll. « Bibliothèque de la Pléiade ».

Kamp Jeannette, Legêne Susan, Van Rossum Matthias, RümKe Sebas, Writing History! A Companion for Historians, traduction de Geschiedenis schrijven. Wegwijzer voor historici, Amsterdam, Amsterdam University Press.

Leroux Robert, History and sociology in France. From scientific history to the Durkheimian school, traduction de Histoire et sociologie en France, New York, Routledge.

Perrot Jean-Claude, Perrot Michelle, Rebérioux Madeleine, Maitron Jean, La Sorbonne par elle-même, édité par Sophie Cœuré, Éditions de la Sorbonne, coll. « Tirés à part ».

STOLLeis Michael, Introduction à l'histoire du droit public en Allemagne, XVI-XXI siècle, traduction d'Aurore Gaillet, Paris, Classiques Garnier.

Thapar Romila, The Historian and her Craft. Collected Essays and Lectures, New Delhi (Inde), Oxford University Press.

Thibaudet Albert, Michelet, édition établie par Stéphane Zékian, Paris, Les Équateurs, coll. « Parallèles ».

Voltaire, CEuvres de 1764-1766, CEuvres complètes de Voltaire, vol. 60B, édition établie par Marie-Hélène Cotoni, Fabrice Brandli et al., Oxford, SVEC.

\section{Figures d'historiens, penseurs de l'histoire}

Abramovici Jean-Christophe, Jounaud Christian (dir.), Michel de Certeau et la littérature, Paris, Centre de recherche historique, coll. «Les dossiers du GRIHL ».

Arlettaz Silvia (dir.), L'Histoire en questions. Mélanges en l'honneur de Mario Turchetti, Paris, Classiques Garnier.

Arnold John H., Hilton Matthew, Rüger Jan (dir.), History after Hobsbawm. Writing the past for the twenty-first century, Oxford, Oxford University Press.

Bellomo Michele (dir.), Studi di storiografia e storia antica. Omaggio a Pier Giuseppe Michelotto, Rome, Arbor Sapientiae.

Boureau Alain, Kantorowicz. Histoires d'un historien, Paris, Les Belles Lettres, coll. « Histoire».

BowIE Ewen (dir.), Herodotus - narrator, scientist, historian, Berlin, De Gruyter.

BRAvo Benedetto, Erodoto sulla Scizia e il lontano Nord-Est. Contributo all'interpretazione del cosiddetto logos scitico, Rome, Edizioni di storia e letteratura.

Brunns Hinnerk, Nettelbeck Joachim, Aymard Maurice (dir.), Clemens Heller, impresario des sciences de l'homme, Paris, Éditions de la Maison des sciences de l'homme. 
Cantillon Alain, Fabre Pierre-Antoine, Rougé Bertrand (dir.), À force de signes. Travailler avec Louis Marin, Paris, Éditions de 1'EHESS, coll. «En temps \& lieux ».

Déruelle Aude, Potin Yann (dir.), Augustin Thierry. L'Histoire pour mémoire, Rennes, PUR, coll. " Histoire ».

DetMer David, Zinnophobia, Winchester (R.-U.), Zero Books.

Doudet Sophie, Madame de Staël, Paris, Gallimard, coll. « Folio biographies ».

Duforx Stéphane, Laval Christian (dir.), Bourdieu et les disciplines, Nanterre, Presses universitaires de Paris Nanterre.

Ferro Marc, Les Ruses de l'histoire. Le passé de notre actualité, entretiens avec Emmanuel Laurentin, Paris, Tallandier.

Garufo Francesco, Morerod Jean-Daniel (dir.), Laurent Tissot, une passion loin des sentiers battus, Neuchâtel, Éditions Alphil.

Georgoudi Stella, Polignac François de (dir.), Relire Vernant, Paris, Les Belles Lettres.

Hau Lisa Irene, Meeus Alexander, Sheridan Brian (dir.), Diodoros of Sicily. Historiographical Theory and Practice in the "Bibliotheke», Louvain, Peeters.

Joly Marc, Pour Bourdieu, Paris, CNRS Éditions.

Kavoulakos Konstantinos, Feenberg Andrew, Georg Lukács's Philosophy of Praxis. From Neo-Kantianism to Marxism, Londres, Bloomsbury Academic.

Löwy Michael, Walter Benjamin : avertissement d'incendie. Une lecture des Thèses «Sur le concept d'histoire » [2014], Paris, Éditions de l'Éclat.

Lussana Fiamma (dir.), Storia e storiografia fra passato e futuro. Scritti in onore di Francesco Barbagallo, Rome, Carocci.

Miltsios Nikos, Tamiolaki Melina (dir.), Polybius and his Legacy, Berlin, De Gruyter.

Quillien Jean, L'Image de Humboldt dans la postérité, Villeneuve-d'Ascq, Presses universitaires du Septentrion.

Roussillon Marine, Guyot Sylvaine, GLYnn Dominic, FragonARd Marie-Madeleine (dir.), Littéraire. Pour Alain Viala, Arras, Artois Presses Université, 2 tomes.

SAWYER Stephen W., Adolphe Thiers. La contingence et le pouvoir, Malakoff, Armand Colin.

ZENKER Nina, Der Breslauer Froissart im Spiegel spätmittelalterlicher Geschichtsauffassung, Petersberg, Michael Imhof.

\section{Épistémologie, méthodologie et didactique de l’histoire}

Anheim Étienne, Le Travail de l'histoire, Paris, Éditions de la Sorbonne, coll. « Itinéraires ».

Beckert Sven, Sachsenmaier Dominic (dir.), Global History, globally. Research and Practice around the World, Londres/Oxford, Bloomsbury Academic.

Besacier Guillaume, Hassoun Mohamed, LArouk Omar, Zreik Khaldoun (dir.), Le Document? Actes du 20 colloque international sur le document numérique (CiDE.20), Paris, Europia productions.

Boucheron Patrick, Hartog François, L'Histoire à venir, Toulouse, Anacharsis. 
Bouton Christophe, Stiegler Barbara (dir.), L'Expérience du passé. Histoire, philosophie, politique, Paris, Éditions de l'Éclat, coll. « Philosophie imaginaire ».

CAIne Barbara, Biography and History, Londres, Palgrave.

Chaubet François (dir.), Faire l'histoire culturelle de la mondialisation, Nanterre, Presses universitaires de Paris Nanterre.

Crimmins Jonathan Mackenzie, The Romantic Historicism to Come, New York, Bloomsbury Academic.

Elovitz Paul H., The Making of Psychohistory. Origins, Controversies, and Pioneering Contributors, New York, Routledge.

Fourquet François, Penser la longue durée. Contribution à une histoire de la mondialisation, Paris, La Découverte.

Gaboriaux Chloé, Skornicki Arnault (dir.), Vers une histoire sociale des idées politiques, Villeneuve-d'Asq, Presses universitaires du Septentrion, coll. «Espaces politiques ».

Hughes-Warrington Marnie, History as Wonder. Beginning with Historiography, Londres, Routledge.

Hunt Lynn, History. Why it matters ?, Cambridge, Polity.

INGLEBERT Hervé, Histoire universelle ou histoire globale? Les temps du monde, Paris, PUF.

Maerker Anna, Sleight Simon, Sutcliffe Adam (dir.), History, Memory and Public Life. The Past in the Present, Londres, Routledge.

Moatti Claudia, Riot-SARcey Michèle (dir.), Pourquoi se référer au passé ?, Ivry-surSeine, Éditions de l'Atelier-les Éditions ouvrières.

Orain Olivier, MARCEL Jean-Christophe (dir.), Penser par écoles, numéro 32 de Revue d'histoire des sciences humaines, Paris, Éditions de la Sorbonne.

Pérez García Manuel, De Sousa Lucio (dir.), Global History and New Polycentric Approaches. Europe, Asia and the Americas in a World Network System, Singapour, Palgrave Macmillan.

Peschanski Denis, Sion Brigitte (dir.), Mémoire et mémorialisation, vol. 2. La Vérité du témoin, Paris, Hermann.

Pinlainen Kalle (dir.), History in the World, Londres, Routledge.

Poe Marshall, How to Read a History Book, Winchester (R.-U.), Zero Book.

Revel Jacques, Un moment, des histoires, postface de Christophe Prochasson, Paris, Éditions de l'EHESS, coll. « Audiographie ».

ZANCARINI-Fournel Michelle, Une histoire nationale est-elle encore possible?, Bordeaux, Presses universitaires de Bordeaux.

\section{L'histoire de l'histoire}

Antoine-Mahut Delphine, ZÉKIAN Stéphane (dir.), Les Âges classiques du XIXe siècle, Paris, Éditions des Archives contemporaines, coll. " Actualité des classiques ».

Binoche Bertrand, Nommer l'histoire. Parcours philosophiques, Paris, Éditions de l’EHESS, coll. « En temps \& lieux». 
Braganca Manu, Louwagie Fransiska (dir.), Ego-histories of France and the Second World War. Writing Vichy, Cham (Suisse), Palgrave Macmillan/Springer.

Cabantous Alain, Les Ombres de Clio. Les nuits historiques existent-elles? XVI ${ }^{e}-\mathrm{XX}^{e}$ siècle, Paris, CNRS Éditions.

Callu Agnès (dir.), Le Mai 68 des historiens, préface de Patrick Boucheron, Villeneuved'Ascq, Presses universitaires du Septentrion.

Charlemagne, les Carolingiens et Metz. Représentation, recomposition et instrumentalisation du passé du Moyen Âge au Xx siècle, Nancy, Presses universitaires de Nancy/ Éditions universitaires de Lorraine.

Cleaver Laura, Worm Andrea (dir.), Writing History in the Anglo-Norman World. Manuscripts, Makers and Readers, c. 1066-c. 1250, Woodbridge, Boydell.

De Cock Laurence, Dans la classe de l'homme blanc. L'enseignement du fait colonial en France des années 1980 à nos jours, Lyon, PUL.

Décultot Élisabeth, Fulda Daniel, Helmreich Christian (dir.), Poetik und Politik des Geschichtsdiskurses. Deutschland und Frankreich im langen 19. Jahrhundert, Heidelberg, Heidelberg Universitätsverlag.

De Francesco Antonino, La Guerre de deux cents ans. Une histoire des histoires de la Révolution française, Paris, Perrin.

Devillers Olivier, Sebastiani Breno Battistin (dir.), Sources et modèles des historiens anciens, Bordeaux/Pessac, Ausonius éditions.

Esteve Cesc (dir.), Disciplining History. Censorship, Theory, and Historical Discourse in Early Modern Spain, Londres, Routledge.

Jurgenson Luba, Werth Nicolas (dir.), Le Goulag. Témoignages et archives, Paris, Robert Laffont, coll. « Bouquins », 2017.

Lacheny Marc, Huftier Arnaud, Amsellem Frédérique, Hincker Louis (dir.), Les Archives familiales des écrivains. Des matériaux, un motif, une question, Valenciennes, Presses universitaires de Valenciennes.

Loskoutoff Yvan (dir.), Héraldique et numismatique: Moyen Âge, Temps modernes. IV, Rouen, Presses universitaires de Rouen et du Havre.

Mambwini Kivuila-Kiaku José, La Conception de l'histoire à Rome chez Salluste, TiteLive et Tacite. Étude littéraire de quelques préfaces, Paris, L'Harmattan.

Martin Jean-Clément, Les Échos de la Terreur. Vérités d'un mensonge d'État, 1794-2001, Paris, Belin.

Matijašić Ivan, Shaping the Canons of Ancient Greek Historiography. Imitation, Classicism, and Literary Criticism, Berlin/Boston, De Gruyter.

Mrrone Martin, Peltz Lucy (dir.), Producing the Past. Aspects of Antiquarian Culture and Practice, 1700-1850, Londres, Routledge.

Piovan Dino, Tucidide in Europa. Storici e storiografia greca nell'età dello storicismo, postface d'Ugo Fantasia, Milan, Mimesis.

RАвіот Jérémie, Écrire, comprendre et expliquer l'histoire de son temps au XIV siècle. Étude des livres XI à XIII de la "Nuova cronica » de Giovanni Villani, Montpellier, Presses universitaires de la Méditerranée, coll. " Histoire et sociétés ».

RIPART Laurent (dir.), Écrire l'histoire, penser le pouvoir. États de Savoie, XVe-XVI siècles, Chambéry, Éditions de l'université de Savoie, coll. " Sociétés, religions, politiques ». 
SANDberg Kaj, Sмith Christopher (dir.), Omnium annalium monumenta. Historical Writing and Historical Evidence in Republican Rome, Leyde/Boston, Brill.

ScHorn Stefan, Studien zur hellenistischen Biographie und Historiographie, Berlin, De Gruyter.

SHeId John, Les Arts de la mémoire et les images mentales, Paris, Publications du Collège de France, coll. "Conférences ».

Turner Mathew, Historians at the Frankfurt Auschwitz Trial. Their Role as Expert Witnesses, Londres/New York, I. B. Tauris.

Wilson Ross J., Natural history. Heritage, Place and Politics, Abingdon, Routledge.

\section{L’histoire à travers les arts, la littérature et les médias}

Actes et artefacts graphiques. Les sens de la préhistoire, numéro 227-228 de L'Homme. Revue française d'anthropologie.

Bouchet Thomas, De colère et d'ennui, Paris, Anamosa.

Cárdenas Bunsen José, La Aparición de los libros plúmbeos y los modos de escribir la historia. De Pedro de Castro al Inca Garcilaso de la Vega, Madrid, Iberoamericana/ Francfort-sur-le-Main, Vervuert.

Cazalas Inès, SARfati LANTER Judith (dir.), Expériences de l'histoire, poétiques de la mémoire. Joseph Conrad, Claude Simon, António Lobo Antunes, Neuilly-sur-Seine, Atlande.

Chamarat-Malandain Gabrielle, Illouz Jean-Nicolas, Labouret Mireille, MarCHAl Bertrand, Scepi Henri, SÉGINGer Gisèle (dir.), Gérard de Nerval, histoire et politique, Paris, Classiques Garnier, coll. « Rencontres ».

Снаssot Joanne, Ghosts of the African Diaspora. Re-Visioning History, Memory, and Identity, Hanover (N.H.), University Press of New England.

Croft Marie-Ange, Gevrey Françoise (dir.), Écrire l'actualité: Edme Boursault spectateur de la cour et de la ville. La Comédie sans titre, Lettres nouvelles, Reims, ÉPURE.

Deperne Marcel, Dichy-Malherme Sarah, Pichard Laëtitia (dir.), Trace(s), Limoges, Presses universitaires de Limoges.

EIROA SAN Francisco Matilde (dir.), Historia y memoria en red. Un nuevo reto para la historiografía, Madrid, Síntesis.

Forget Philippe, Pesnel Stéphane (dir.), Joseph Roth. L'exil à Paris, Rouen, Presses universitaires de Rouen et du Havre.

Gobille Boris, Le Mai 68 des écrivains. Crise politique et avant-garde littéraire, Paris, CNRS Éditions.

Grande Nathalie, Pierre Chantal (dir.), Légendes noires, légendes dorées, ou comment la littérature fabrique l'histoire (XVII ${ }^{e}$ XIX ${ }^{e}$ siècle), Rennes, PUR.

Guo Rong, Approaching History. The Fictional Worlds of Ha Jin and Yan Geling, Paris, Honoré Champion, coll. « Bibliothèque de littérature générale et comparée ».

HAMEL Jean-François, LEFORT-FAVREAU Julien (dir.), Écritures de la contestation. La littérature des années 68, numéro 54/1 d'Études françaises. 
Hersant Marc, Jomand-Baudry Régine (dir.), Conte et histoire (1690-1800), Paris, Classiques Garnier, coll. « Rencontres ».

Houssais Yvon (dir.), Fictions médiévales pour la jeunesse, Besançon, Presses universitaires de Franche-Comté.

KLEIN Richard (dir.), À quoi sert l'histoire de l'architecture aujourd'hui ?, postface d'Emmanuel Laurentin, Paris, Hermann.

MaIRA Daniel, Renaissance romantique. Mises en fictions du XVI siècle (1814-1848), Genève, Droz.

Pernot Denis, Henri Barbusse : les discours du «Feu », Dijon, Éditions universitaires de Dijon.

PrÉvost Maxime, Alexandre Dumas mythographe et mythologue. L'aventure extérieure, Paris, Honoré Champion, coll. « Romantisme et modernités ».

Roulin Jean-Marie, Saminadayar-Perrin Corinne (dir.), Fictions de la Révolution, Rennes, PUR.

Schafer Valérie (dir.), Temps et temporalités $d u$ Web, Nanterre, Presses universitaires de Paris Nanterre.

Simard-Houde Mélodie, Le Reporter et ses fictions. Poétique historique d'un imaginaire, Limoges, Presses universitaires de Limoges.

Wattel Anne, Robert Merle. Écrivain singulier du propre de l'homme, Villeneuved'Ascq, Presses universitaires du Septentrion.

WaWrzyniak Natalia, Lamentation et polémique au temps des guerres de Religions, Paris, Classiques Garnier, coll. « Bibliothèque d'histoire de la Renaissance ».

ZInk Michel, Ce que nous devons aux anciens poètes de la France, leçon de clôture prononcée le 10 février 2016, Paris, Éditions du Collège de France, coll. «Leçons de clôture ». 